\title{
Charged black holes in Hořava gravity
}

\section{Stefan Janiszewski, Andreas Karch, Brandon Robinson and David Sommer}

Department of Physics, University of Washington,

Seattle, WA, 98195-1560, U.S.A.

E-mail: stefanjj@uw.edu, akarch@uw.edu, robinb22@uw.edu, dsommer2@uw.edu

ABSTRACT: We explore static spherically symmetric black hole solutions allowing a bulk $\mathrm{U}(1)$ vector field in the khronometric formulation of Hořava gravity by way of EinsteinÆther. We examine analytic solutions and study numerical results in the limit that the khronon does not backreact on the metric.

KEYwords: AdS-CFT Correspondence, Black Holes

ArXiv EPrint: 1401.6479 


\section{Contents}

1 Introduction 1

2 Khronons and universal horizons $\quad 2$

2.1 Asymptotically hyperbolic results 4

2.2 Probe limit 5

3 Charged black holes in khronometric Hor̆ava gravity 6

3.1 Probe limit 6

3.2 Analytic solutions 8

4 NR holography on the $\mathrm{AdS}_{2}-12$

5 Conclusion $\quad 14$

\section{Introduction}

There has recently been increased interest in a particular theory of gravity that manifestly breaks Lorentz invariance and is power counting renormalizable: Hořava gravity [1]. One of the attractive features of Hořava gravity is that even in the absence of matter it allows solutions with anisotropic scaling between space and time i.e., $t \rightarrow \lambda^{z} t$ and $x_{i} \rightarrow \lambda x_{i}$, where $z$ is the dynamical critical exponent, even in the low energy limit where only two-derivative terms are kept in the action. The degrees of freedom are the familiar quantities of the ADM formulation of GR: the lapse $N$, shift vector $N_{I}$, and spatial metric $G_{I J}$. The realization of solutions with Lifshitz scaling provide another possible utilization of holography that capture features common to condensed matter systems [2].

One of the most interesting questions in Hořava gravity, as well as similar nonrelativistic constructions as summarized in [3], is to what extent horizons and their thermodynamic properties, which are such a paradigmatic feature of relativistic gravity, continue to exist in the non-relativistic setting. Our main tool to address these questions is the equivalence between the low energy limit of Hořava and another well studied theory of gravity endowed with a preferred notion of time, Einstein-Æther (E- $Æ)$. The nature of the equivalence was argued in [4] to arise in static, spherically symmetric geometries. It was also recently conjectured in [5] that the correspondence between Hořava gravity and E- $\mathbb{E}$ runs deeper. The author of [5] argues that in the limit that the coupling of the 'twist' of

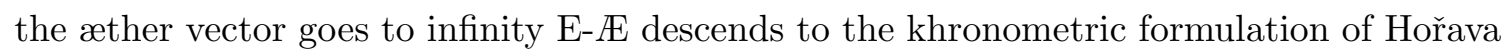
gravity, and one can also find solutions of the khronometric theory that are not hypersurface orthogonal Einstein-Æther solutions e.g., slowly rotating black holes. Here, however, we will focus on static, spherically symmetric geometries equipped with a preferred notion 
of time that allows a geometric construction of both theories based on leaves of constant global time foliating the space-time [3]. Transformations that preserve the global time coordinate are good symmetries of the two theories. In Hořava and Einstein-Æther, we have foliation preserving diffeomorphisms (Fdiffs) that include time reparameterizations $t \rightarrow \tilde{t}(t)$ and spatial diffeomorphisms $x_{I} \rightarrow \tilde{x}_{I}(x)$. In general in Hořava gravity, time dependent spatial diffeomorphisms are allowed [3].

Importantly, the Hořava and E- $Æ$ equivalence affords a window into interesting solutions realizing causal horizons in a natural way. Since Lorentz invariance is broken, there can be modes propagating faster than the speed of light $c$, which means that light cones are no longer the objects encoding causality. Instead, causality is enforced by the requirement that propagation is unidirectional into the future according to the preferred time. Recent work in [6] has shown that the foliation naturally captures causal horizons as the boundary surface at timelike infinity. This surface is called the "universal horizon" as it represents a trapping surface for arbitrarily fast propagating modes.

This leads one naturally to ask how universal these horizons are; do they appear outside of the asymptotically flat backgrounds; do they persist with the inclusion of parameters beyond mass characterizing the solution e.g., charge, angular momentum? The work done in [7] has shown that universal horizons do exist in asymptotically Anti-de Sitter (AdS) spacetimes. The realization of universal horizons in the probe limit lead to the search for analytic solutions realizing the same behavior. Recently, such solutions were constructed systematically in [7].

The primary purpose of this paper is to explore more general geometrical settings. Can we successfully identify a causal boundary in the probe limit with the inclusion of electric charge on the horizon? Can we then turn on finite couplings and find black hole solutions in the fully backreacting theory? Can we determine consistently the thermodynamics of such solutions? Is there a way to realize a holographic picture on the resulting background, and can we interpret the results using language familiar to us for relativistic backgrounds?

In section 2, we will discuss the relationship between spherically symmetric EinsteinÆther and the low energy Hořava theories. In addition, we will review the results obtained for the asymptotically flat and AdS cases. In section 3, we will test the universality of the appearance of the causal boundary in an AdS-Reissner-Nördstrom background and discuss the subtleties as the charge is increased toward extremal values. We will then obtain an analytic solution for charged backgrounds, attempting both asymptotically AdS and flat geometries, and examine the resulting near-horizon structure. Lastly in section 4, we will compute the two point correlation of non-relativistic charged scalars in the nearhorizon geometry (e.g., $\mathrm{AdS}_{2} \times R^{2}$ ) and attempt an interpretation. In particular, we will compare those results to those of the relativistic charged scalars using the $\mathrm{AdS}_{2} / C F T_{1}$ correspondence.

\section{Khronons and universal horizons}

Here we offer a brief overview of the relevant concepts that will be useful for the following sections. For a comprehensive look at the khronon formalism and its uses see $[2,4,7,8]$. 
As mentioned above, we can understand the low energy effective Hořava theory in a purely geometric way. The degrees of freedom available to us in a theory with Fdiff invariance come in the form of the lapse $N$, shift vector $N_{I}$, and spatial metric $G_{I J}$ familiar in the ADM decomposition of the metric in standard GR,

$$
d s^{2}=-N^{2} d t^{2}+G_{I J}\left(d x^{I}+N^{I} d t\right)\left(d x^{J}+N^{J} d t\right) .
$$

The low energy Hořava action is

$$
I_{H}=\frac{1}{16 \pi G_{H}} \int \mathrm{d} t \mathrm{~d}^{3} x N \sqrt{G}\left(K_{I J} K^{I J}-(1+\tilde{\lambda}) K^{2}+(1+\tilde{\beta})(R-2 \Lambda)+\tilde{\alpha} \frac{\nabla_{I} N \nabla^{I} N}{N^{2}}\right),
$$

where $K_{I J}=\frac{1}{2 N}\left(\partial_{t} G_{I J}-\nabla_{I} N_{J}-\nabla_{J} N_{I}\right)$ is the extrinsic curvature of the spatial leaves foliating the spacetime, $G$ is the determinant of the spatial metric, and $\Lambda$ is the cosmological constant. The couplings $(\tilde{\alpha}, \tilde{\beta}, \tilde{\lambda})$ enter due to the Fdiff symmetry, and $G_{H}$ sets the Planck mass.

Fdiffs allow for time reparameterizations, $t \rightarrow \tilde{t}(t)$, which lets us rewrite the low energy Hořava gravity action in a covariant form by way of a scalar field, $\phi(t)$ called the khronon,

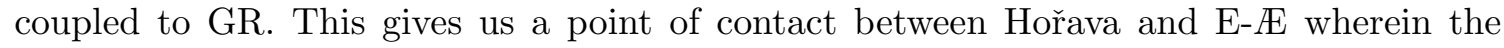
æther vector, $u^{M}$ can be written in terms of the khronon:

$$
u_{M}=\frac{-\partial_{M} \phi}{\sqrt{-g^{P Q} \partial_{P} \phi \partial_{Q} \phi}} .
$$

$u_{M}$ is a time-like unit vector normal to the spatial leaves of the foliation. By making this identification, and an appropriate rescaling and renaming of couplings, (2.2) can be recast as

$$
I_{k h}=\frac{1}{16 \pi G_{k h}} \int \mathrm{d}^{4} x \sqrt{-g}\left(R-2 \Lambda+c_{4} u^{M} \nabla_{M} u^{N} u^{P} \nabla_{P} u_{N}-c_{2}\left(\nabla_{M} u^{M}\right)^{2}-c_{3} \nabla_{M} u^{N} \nabla_{N} u^{M}\right)
$$

with the following relations

$$
\frac{G_{H}}{G_{k h}}=1+\tilde{\beta}=\frac{1}{1-c_{3}}, \quad \tilde{\alpha}=\frac{c_{4}}{1-c_{3}}, \quad 1+\tilde{\lambda}=\frac{1+c_{2}}{1+c_{3}} .
$$

After finding the desired solutions, we will need to map to the adapted coördinates of the $\mathrm{ADM}$ form of the metric to find the Hořava degrees of freedom.

The speed for the scalar mode, $s_{0}$, and the spin- 2 graviton $s_{2}$ can be determined from the linearized theory around a flat background in the weak field, low speed limit:

$$
s_{2}^{2}=\frac{1}{1-c_{3}}, \quad s_{0}^{2}=\frac{\left(c_{2}+c_{3}\right)\left(D-1+c_{4}\right)}{c_{4}\left(1-c_{3}\right)\left(D-1+D c_{2}+c_{3}\right)} .
$$

In the probe limit, which we will be considering in the next section, the couplings are taken to small values $c_{i} \ll 1$, and the speed of the two present modes become

$$
s_{2}^{2} \approx 1, \quad s_{0}^{2} \approx \frac{c_{2}+c_{3}}{c_{4}} .
$$




\subsection{Asymptotically hyperbolic results}

The work in [7] is instructive to understand as it provides a basis for further exploration in charged backgrounds. The primary goal in [7] was to realize black hole solutions to (2.4) that had an asymptotic geometry amenable to holographic descriptions [2]. That is, far from the interior of the $d+1$ dimensional space-time (i.e., as $r \rightarrow 0$ ) the metric has the form

$$
d s^{2} \approx-\left(\frac{L^{2}}{r^{2}}\right)^{z} d t^{2}+\frac{L^{2}}{r^{2}}\left(d r^{2}+d \vec{x}^{2}\right),
$$

where $d \vec{x}^{2}$ is a section on the $d-1$ dimensional plane. For the rest of the paper we will be using $d=3$.

The translational invariance on the spatial section indicates that we will be looking for planar horizon solutions (black branes). Working in the Poincaré-like coördinates above lends itself to numerical analysis near the boundary, which will prove useful in the probe limit calculations. Further, it is more natural to use a coördinate system that covers the whole manifold of a black hole space-time such as in-falling Eddington-Finkelstein coördinates (IEF). This choice will again give a more natural setting in which to perform the probe calculations. In IEF, we define the in-falling time $v=t-r^{*}$ where the tortoise coördinate $r^{*}=\int \mathrm{d} r \sqrt{-\frac{g_{r r}}{g_{t t}}}=\frac{L}{z}\left(\frac{r}{L}\right)^{z}$ such that, setting $L=1$, the new metric is

$$
d s^{2}=-\frac{d v^{2}}{r^{2 z}}-\frac{2}{r^{z+1}} d v d r+\frac{d \vec{x}^{2}}{r^{2}} .
$$

This provides us with the asymptotic form of the generic ansatz for a spherically symmetric black hole space-time,

$$
d s^{2}=-e(r) d v^{2}-2 f(r) d v d r+\frac{d \vec{x}^{2}}{r^{2}} .
$$

Now that we have in-hand an ansatz for the metric, we need to take care of the æther vector, which asymptotically should reproduce Poincaré time $(\phi \sim t)$ of eq. (2.8). In IEF this requires,

$$
u_{M}=\left(-\frac{1}{r^{z}},-\frac{1}{r}, 0,0\right),
$$

for the generic timelike unit vector respecting the same symmetries

$$
u_{M}=\left(-\frac{a(r)^{2} e(r)+f(r)^{2}}{2 a(r) f(r)},-a(r), 0,0\right) .
$$

Since we are respecting spherical symmetry in $d=3$, we can note that hypersurface orthogonality demands the curl of $u_{M}$ given by $\omega_{M}=\epsilon_{M N P Q} u^{N} \nabla^{P} u^{Q}$ vanishes. We can then rewrite the $c_{4}$ term in eq. (2.4) as

$$
u^{M} \nabla_{M} u^{N} u^{P} \nabla_{P} u_{N}-\omega_{M} \omega^{M}=-\frac{1}{2} F_{M N} F^{M N},
$$

with $F_{M N}=\partial_{M} u_{N}-\partial_{N} U_{M}$ playing the role of a 'field strength'. The zeroth order solution to the equations of motion, found by varying with respect to $e(r), f(r)$, and $a(r)$ and expanding each as their asymptotic forms multiplied by a power series in $r$, necessitates $c_{4}=\frac{z-1}{z}$ and, in arbitrary $(d+1)$ dimensions, $\Lambda=-\frac{1}{2}(z+d-2)(z+d-1)$. For example, the asymptotically $\mathrm{AdS}_{4}$ solution is given by $z=1, c_{4}=0$, and $\Lambda=-3$. 
In making the asymptotic expansions of the metric functions and the khronon, we can solve for the coefficients in the expansions order by order in the hope to obtain an analytic expression for $e(r)$ and $f(r)$. Specifically, we would hope that the expansions for the metric functions terminate at some order and leave us with a small number of constants describing the solution. At the very least, we would desire an expansion that has an obvious analytic structure. Indeed, it was found that for $z=1$, the expansions for both $e(r)$ and $f(r)$ terminate quickly, at least in checking to $\mathcal{O}\left(r^{30}\right)$, while $a(r)$ does not but is controlled by two parameters $C_{e}$ and $C_{a}$. The appearance of two parameters is troubling at first, but when one imposes regularity at the sound horizon of the scalar mode, one parameter is immediately fixed. Once the truncated functions are in hand, we will make the ansatz $a(r)=-\frac{1}{r} b(r)$, where the asymptotic behavior $-\frac{1}{r}$ is multiplying an analytic function $b(r)$. This assumption of the analyticity of $b(r)$ does not always produce the correct results in the asymptotically Lifshitz, $z \neq 1$, solutions as discussed in [7]. However for the asymptotically AdS black hole, the metric functions and the khronon can be solved exactly and adapted to the ADM-like coördinates of Hořava gravity to yield

$$
d s^{2}=-\frac{\left(r^{3}-r_{*}^{3}\right)^{2}}{r^{2} r_{*}^{6}} d t^{2}+\frac{r_{*}^{6}}{r^{2}\left(r^{3}-r_{*}^{3}\right)^{2}}\left(d r+\frac{r^{3}\left(r^{3}-r_{*}^{3}\right)}{\sqrt{1-c_{3} r_{*}^{6}}} d t\right)^{2}+\frac{d \vec{x}^{2}}{r^{2}} .
$$

\subsection{Probe limit}

While the analytic solution found in the previous section is interesting in its own right, the probe limit offers us the opportunity to study Hořava gravity using GR solutions as backgrounds for the khronon. That happens because in the probe limit the couplings that control the dynamics of the khronon are parametrically small, and so metrics that solve the Einstein equations, solve the gravitational equations of motion for Hořava gravity due to the lack of backreaction. The khronon simply imprints a preferred notion of time on a given solution of Einstein gravity. The result is needing to solve, numerically in most cases, the equations of motion for the scalar khronon on a known background. To that end, we will quote the method used in [7] and the results obtained therein for future use in guiding us with the inclusion of charge.

To begin, we can see that in the limit of parametrically small couplings, we can use as our background for the asymptotically AdS solution the metric for an AdS-Schwarzschild black brane with unit mass,

$$
d s^{2}=\frac{1}{r^{2}}\left(-\left(1-r^{3}\right) d v^{2}-2 d v d r+d \vec{x}^{2}\right) .
$$

The æther vector, being a unit timelike vector, in these IEF coördinates then can be taken to have the form (akin to eq. (2.12))

$$
u_{M}=\left(-\frac{1+h(r)}{2 r} \sqrt{\frac{1-r^{3}}{h(r)}},-\sqrt{\frac{h(r)}{r^{2}\left(1-r^{3}\right)}}, 0,0\right) .
$$

The demand that asymptotically the khronon encodes Poincaré time requires $h(0)=1$ and thus $\left.u_{M}\right|_{r \rightarrow 0} \rightarrow\left(-\frac{1}{r},-\frac{1}{r}, 0,0\right)$. The khronon action is then

$$
I_{k h}=\frac{-c_{4}}{16 \pi G_{K}} \int \mathrm{d} v \operatorname{rd} x^{2} \sqrt{-g}\left(\frac{1}{2} F_{M N} F^{M N}+s_{0}^{2}\left(\nabla_{M} u^{M}\right)^{2}\right) .
$$


It is straightforward to find the equations of motion for $h(r)$, the result is a non-linear ODE. Numerical integration starts by Taylor expanding around the value of the radial coördinate $r_{c}$ at which $h\left(r_{c}\right)=\frac{1-s_{0}}{1+s_{0}}$ for fixed scalar speed $s_{0}^{2}$. A shooting method can then be implemented to determine exactly which value of $r_{c}$ corresponds to the correct boundary conditions at $r=0$. The value $r_{c}$ labels the location of the sound horizon for the scalar mode [6]. It is clear that when the speed of the scalar and spin-2 graviton coincide the $r_{c}$ associated with the scalar mode will match that of the metric horizon, which is the sound horizon of the spin-2 mode.

After finding the location of the sound horizon for a given $s_{0}^{2}$, numerical integration can be performed from $r_{c}$ toward the interior $(r \rightarrow \infty)$ to determine the location of any singular surfaces. For $s_{0}^{2}<1$, the sound horizon sits outside of the metric horizon $\left(r_{c}<r_{h}\right)$, and for $s_{0}^{2}>1$, the sound horizon is interior to the metric horizon. One must take care as the position of the metric horizon is a singular point of the equations of motion and so must be bridged by matching a Taylor series both on the interior and exterior plus/minus some infinitesimal distance.

\section{Charged black holes in khronometric Hořava gravity}

Once the metric horizon has been traversed, we can continue integrating to the interior in search of the position of the universal horizon $r_{*}$, which is seen when the EddingtonFinklestein time component of the æther vector, $u_{v}$, vanishes. In the language of Horava gravity, $r_{*}$ is the location at which the lapse vanishes and the leaves of the foliation 'pile up', e.g., $h(r)=-1$. This behavior is illustrated quite dramatically in figure 1, showing the Penrose diagram of AdS-Schwarzschild space foliated by the preferred time of Hořava gravity. It is apparent that there exists a radial coördinate beyond which there is no causal connection to the exterior space no matter the speed of propagation and, as discussed earlier, is given by the leaf of the foliation at timelike infinity. Given the success in finding universal horizons in the probe limit of an AdS-Schwarzschild black brane and asymptotically AdS solutions to the full backreacting theory, we can begin to ratchet up the electric charge. The hope would be to find that the causal boundaries previously discovered do occur generically in the probe limit. In addition, we can consider finite values of the couplings and hopefully find interesting backreacted asymptotically AdS charged solutions.

\subsection{Probe limit}

The analysis of the AdS-Schwarzschild black brane carries over to the AdS-ReissnerNördstrom background in the probe limit with the obvious additional parameter of charge, $Q$. With this additional parameter and knowledge of its problematic limits from literature on charged black holes as GR solutions, it would be wise to fully explore the range of charge from $Q=0$, which will hopefully reproduce results in the previous section, all the way up to extremality where the inner and outer horizons coalesce. Since we too desire to study the behavior of the khronon on a geodesically complete manifold, it is instructive to consider first the AdS-Reissner-Nördstrom metric in the in-falling Eddington-Finklestein 


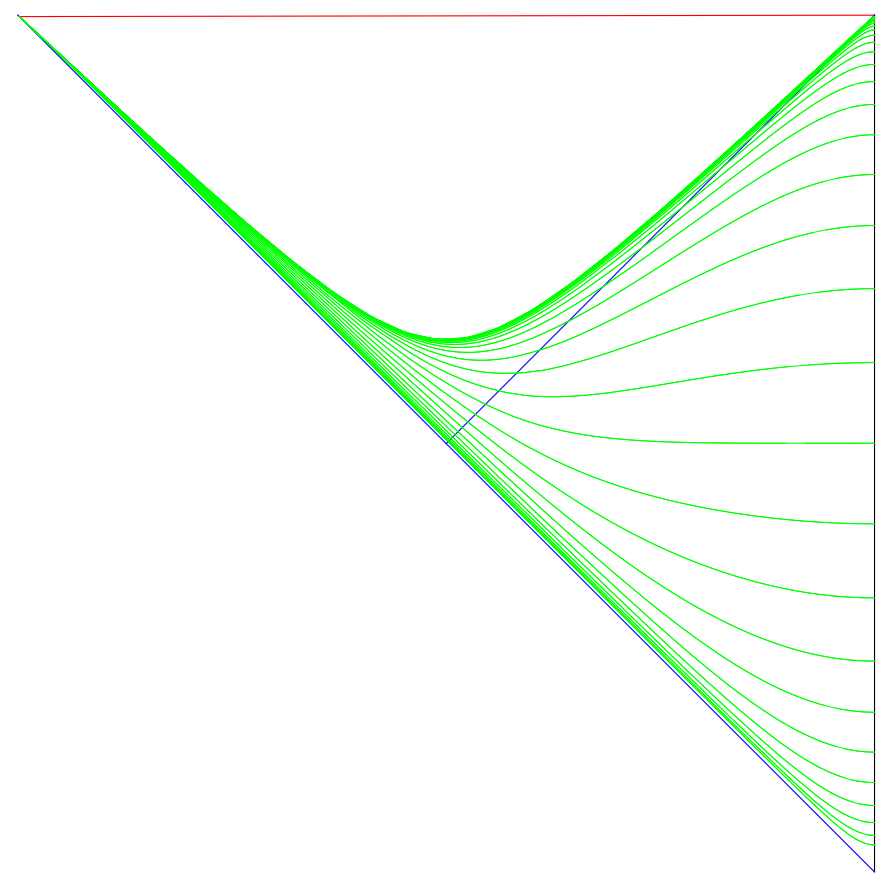

Figure 1. Foliation of the AdS-Schwarzschild black hole by leaves of constant $u^{\mu}$ (green lines). The vertical black line represents the AdS boundary. The horizontal red line shows the singularity, and the blue lines at $45^{\circ}$ represent the sound horizon of the spin- 2 metric degrees of freedom.

coördinates:

$$
d s^{2}=\frac{1}{r^{2}}\left(-\left(1-M r^{3}+Q r^{4}\right) d v^{2}-2 d v d r+d \vec{x}^{2}\right)
$$

where $M$ and $Q$ represent the mass and charge, respectively, of the black brane.

The æther vector in terms of the scalar khronon, $\phi(r)$, for the AdS-Reissner-Nördstrom background is:

$$
u_{M}=\left(-\frac{1+\phi(r)}{2 r \sqrt{\frac{\phi(r)}{1-M r^{3}+Q r^{4}}}},-\sqrt{\frac{\phi(r)}{r^{2}\left(1-M r^{3}+Q r^{4}\right)}}, 0,0\right) .
$$

On this background the probe action for the khronon takes the form,

$$
I_{k h M}=\frac{-1}{16 \pi G_{K}} \int \mathrm{d} t \mathrm{~d}^{d} x \sqrt{-g}\left(F^{M N} F_{M N}+s_{0}^{2}\left(\nabla_{N} u^{M}\right)^{2}+b^{2} R_{M N} u^{M} u^{N}\right)
$$

where $F_{M N}=\partial_{M} u_{N}-\partial_{N} u_{M}$. The $R_{M N} u^{M} u^{N}$ term, while not manifestly zero as in flat backgrounds, does not contribute to the khronon equations of motion. Varying with respect to $\phi(r)$ we can make a series expansion around the sound horizon $\phi\left(r_{c}\right)=\phi_{c}$ and solve the equations of motion order by order in $\left(r-r_{c}\right)$ as in the analysis of the AdS-Schwarzschild black brane.

Thus, the primary result of the numerical analysis is that as the inner and outer horizons coalesce as $Q \rightarrow Q_{c}=3^{1 / 3}\left(\frac{3 M}{4}\right)^{4 / 3}$ the universal horizon is being pinched between the two surfaces. We can see this clearly from figure 2 that as we approach criticality, 


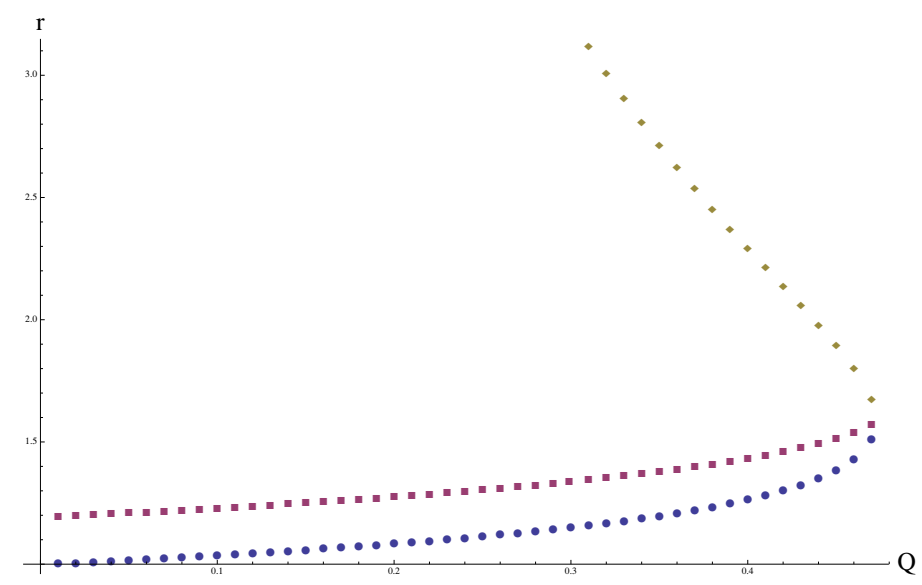

Figure 2. Plot of the value of the different horizon radii as we change the charge in the background up to near the critical value $Q_{c}$. Upper $(\diamond)$ curve represents $r_{h}^{-}$, the center $(\square)$ curve shows $r_{*}$, and the lower $(\bigcirc)$ curve displays $r_{h}^{+}$. One can see that the curves coalesce as $Q \rightarrow Q_{c}$.

$r_{h} \approx 1.5863, r_{*}$ is dragged toward this location. Upon constructing the Penrose diagrams, figure 3 and figure 4, as in the AdS-Schwarzschild case, we can see that the leaves of the foliation are piling up nearer to the metric horizon as Q increases. This observation will play a role in searching for analytic black hole solutions in the next section as we will see this behavior manifest itself in a natural way.

\subsection{Analytic solutions}

Taking the couplings away from the probe limit to finite values, we want to consider the following action for full backreacting khronometric theory

$$
\begin{aligned}
& I_{k h}=\frac{1}{16 \pi G_{K}} \int \mathrm{d} t \mathrm{~d}^{3} x \sqrt{-g}( R-2 \Lambda-\frac{c_{4}}{2} F_{a b} F^{a b}-c_{2}\left(\nabla_{b} u^{a}\right)^{2}-c_{3} \nabla_{b} u^{a} \nabla_{a} u^{b} \\
&\left.+\mu \mathcal{F}_{a b} \mathcal{F}^{a b}+\frac{\kappa}{4} u^{a} \mathcal{F}_{a b} u^{c} \mathcal{F}_{c}^{b}\right)
\end{aligned}
$$

where $F_{a b}$ is again the khronon "field strength", $\mathcal{F}_{a b}=\partial_{a} \mathcal{A}_{b}-\partial_{b} \mathcal{A}_{a}$ is the electromagnetic field strength, and $\mathcal{A}_{t}=\rho(r)$ is the gauge potential. Traditionally, we would only have $\mathcal{F}^{2}$ in considering the contribution of the Maxwell field, but owing to less restrictive FDiff symmetry, we have the presence of the novel $u^{a} \mathcal{F}_{a b} u^{c} \mathcal{F}_{c}{ }^{b}=E^{2}$. When we consider asymptotically flat solutions, $\mathcal{F}^{2}$ does not play as an important role as it does for asymptotically AdS and will then be disregarded. Analysis was done to account for the possibility of dyonic black holes with the $\sqrt{-g} \mu \mathcal{F}_{a b} \mathcal{F}^{a b}$ included in (3.4) and $\mathcal{A}_{i} \neq 0$ but no fruitful results appeared.

In this section, we will use as our metric ansatz eq. (2.10) giving the asymptotic geometry (2.8) with $z=1$. We also must specify the asymptotic behavior of $\rho(r)$, which simply amounts to solving Maxwell's equations in $\mathrm{AdS}_{4}$ i.e., $\rho(r) \sim Q r$ for small $r$. With the asymptotic expansions for $e(r), f(r)$, and $a(r)$ from eq. (2.1), we can solve the equations of motion order by order to $\mathcal{O}\left(r^{30}\right)$ noting that for $z=1$ the zeroth order requires $\alpha=0$ 


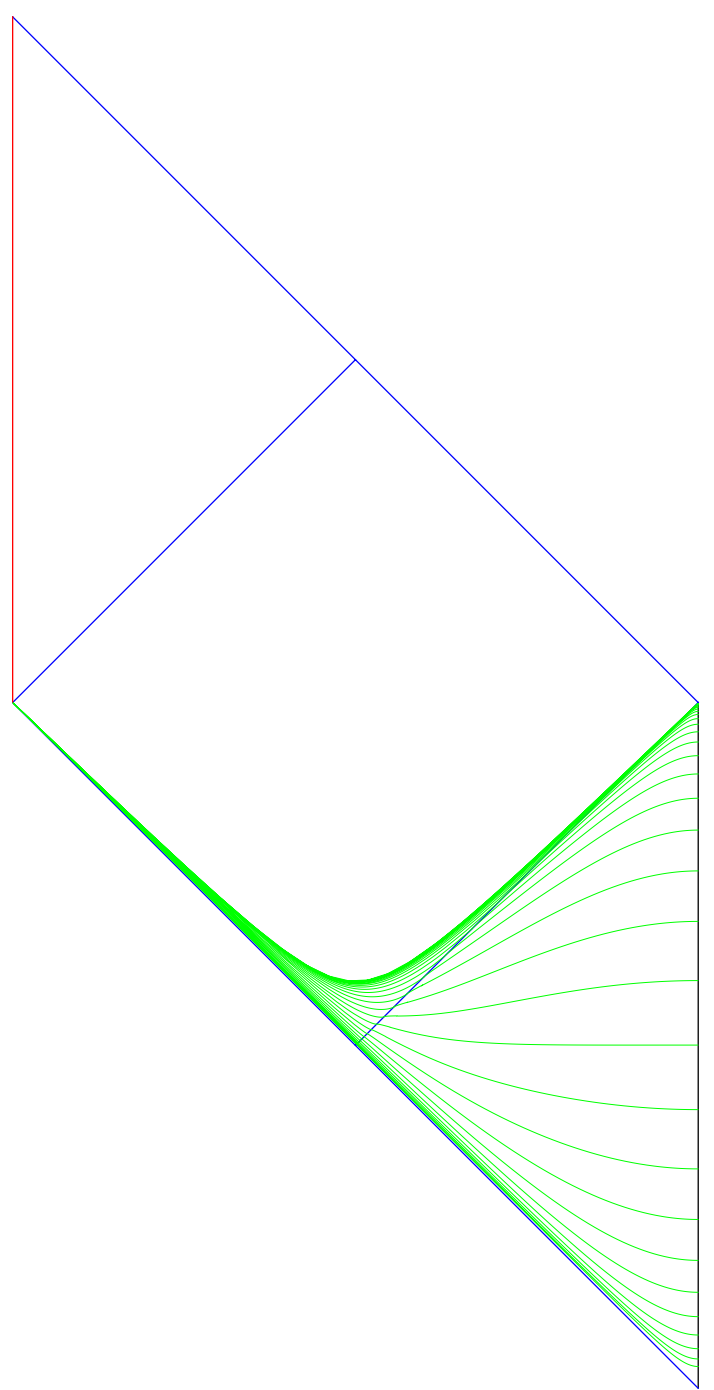

Figure 3. Foliation, spatial profile of constant preferred time, of the AdS-RN background with $M=1$ and $Q=0.25$.

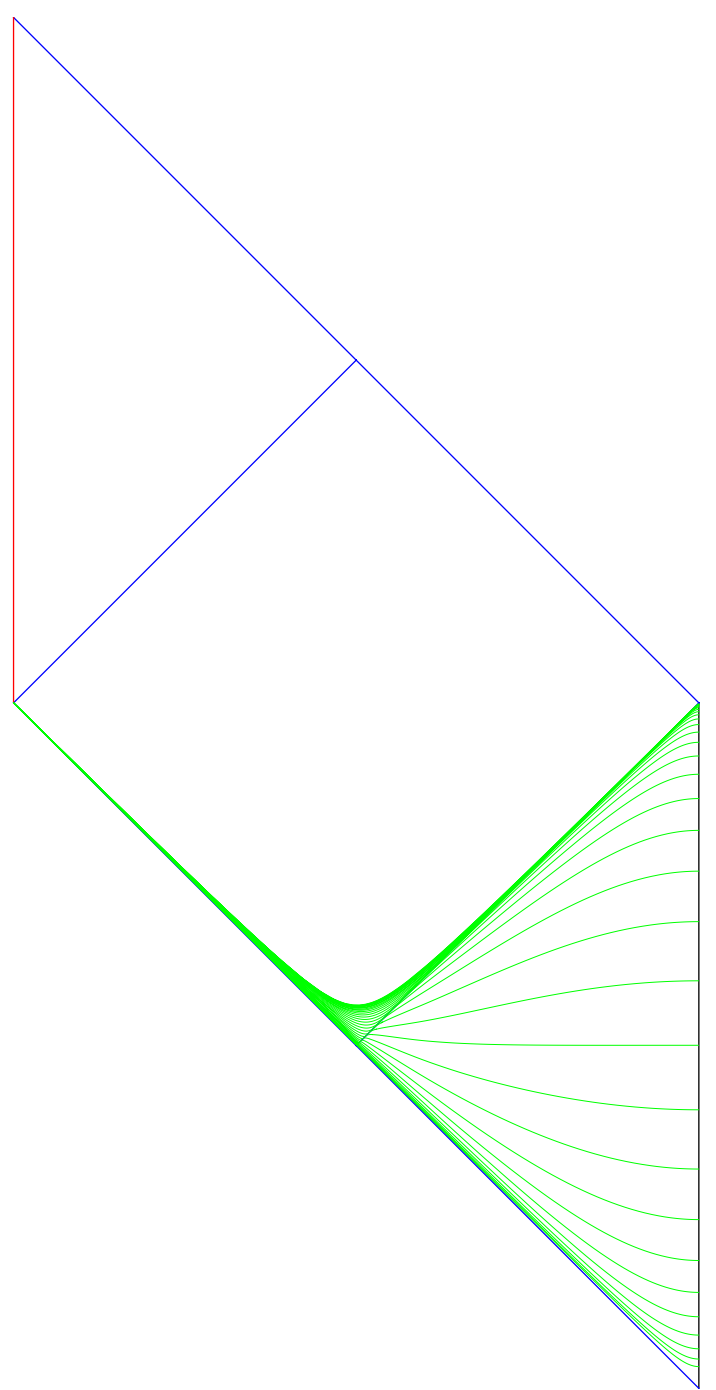

Figure 4. Foliation of AdS-RN background with $M=1$ and $Q=0.4$.

and $\Lambda=-3$, which results in

$$
\begin{aligned}
& e(r)=\frac{1}{r^{2}}\left(1-2 r^{3} C_{a}+\frac{\kappa-8 \mu}{24} C_{e} r^{4}\right) \\
& f(r)=\frac{1}{r^{2}} \\
& a(r)=-\frac{1}{r}\left(1+C_{a} r^{3}+\frac{1}{2}\left(3 C_{a}^{2}-C_{e}\right) r^{6}+\frac{1}{2}\left(5 C_{a}^{2}-3 C_{e}\right)+\ldots\right) \\
& \rho(r)=\sqrt{C_{e}} r .
\end{aligned}
$$

We can see that the metric functions and the scalar potential truncate, while the khronon function $a(r)$ does not. We can appeal to the logic for the uncharged case in using the above expressions as ansatze in our equations of motion with $a(r)=-\frac{1}{r} b(r)$ where $b(r)$ 
is an analytic function. Solving the resulting differential equation coming from the $e(r)$ equations of motion for $b(r)$ and fixing the constants by comparing to the $a(r)$ series gives

$$
b(r)= \pm\left(1-2 C_{a} r^{3}+C_{e} r^{4} \frac{\kappa-8 \mu}{24}\right)^{-\frac{1}{2}}
$$

where the + sign is taken for $C_{a}>0$ and the - sign for $C_{a}<0$. Inserting eq. (3.5) in the equations of motion, we find satisfaction only when $\kappa=-8 \mu$. However, this solution does not descend smoothly to a probe limit solution, except for the extremal case, and is thus disregarded. Therefore, non-extremal AdS-Reissner-Nördstrom is not a physical solution of eq. (3.4), as already seen in the probe limit of section 3.1.

While we only have probe limit solutions with asymptotically AdS geometry, we can still search for asymptotically flat analytic solutions. We cannot make a metric ansatz with a planar geometry on the horizon in this case, but instead we will assume the metric has the form

$$
d s^{2}=-e(r) d v^{2}-2 f(r) d v d r+1 / r^{2}\left(d \theta^{2}+\sin ^{2}(\theta) d \phi^{2}\right),
$$

and the æther vector has the form eq. (2.12), where asymptotically

$$
e(r) \sim 1, \quad f(r) \sim \frac{1}{r^{2}} \quad a(r) \sim-\frac{1}{r^{2}} \quad \rho(r) \sim Q r .
$$

Inserting eq. (3.6) into eq. (3.4) and applying the same asymptotic prescription to the resulting equations of motion, we find

$$
\begin{aligned}
& e(r)=1-2 C_{a} r+\left(C_{a} r\right)^{2} \\
& f(r)=\frac{1}{r^{2}} \\
& a(r)=1-C_{a} r+C_{a}^{2} r^{2}-C_{a}^{3} r^{3}+\ldots \\
& \rho(r)=2 r \sqrt{\frac{(2-\alpha) C_{a}^{2}}{\kappa}} .
\end{aligned}
$$

We can immediately recognize the above solution as the extremal Reissner-Nördstrom black hole with the khronon encoding global time. The latter point comes from formally taking the sum for $a(r)=\frac{1}{1-C_{a} r}=\sqrt{e(r)^{-1}}$. One should note that there was no specification of the couplings appearing in eq. (3.4), which is a departure from the uncharged asymptotically AdS solutions. Further, there is a lone parameter describing our black hole, which is easily seen to be the location of both the metric and universal horizon. This should be expected from the nature of the behavior of the universal horizon near extremal charge in the probe limit.

In transforming back to the adapted coördinates for Hořava gravity, we find no surprises. That is, the shift vector $N_{I}=0$, the lapse $N=\sqrt{-g_{t t}}$, and the spatial metric is just the induced metric on a slice of constant time. This stands in contrast to the asymptotically AdS solution in section 2.1 that had a far more interesting structure. Thus, we have a perfectly good relativistic vacuum as a solution to our non-relativistic theory.

While the full asymptotically flat geometry is not useful in the context of holography, it is well known that for the extremal Reissner-Nördstrom the near horizon geometry is 
that of $\mathrm{AdS}_{2} \times S^{2}$. This is the setting in which we will calculate correlation functions. It is important then to understand the thermodynamics as we approach the horizon. So, it is important to understand the thermodynamics of the space-time in both limits. However, the calculations for the global geometry are trivial as the matter content (i.e., the khronon) does not contribute to the calculation of the temperature or free energy, and we simply recover well known results for the extremal Reissner-Nördstrom black hole. There was some hope that the near horizon geometry would remember something about the free energy outside of the throat, but we found what one would expect for a vacuum space-time like $\mathrm{AdS}_{2} \times S^{2}$ : zero temperature and zero free energy.

As we have found that the near horizon geometry is of particular interest, it is natural to check whether we can find $\mathrm{AdS}_{2} \times \mathcal{M}^{2}$ to be a global solution of the equations of motion derived from eq. (3.4) for $\mathcal{M}^{2}$ being $R^{2}, S^{2}$, or $H^{2}$, that is, a plane, a sphere, or a hyperbolic plane, respectively. We note that $\mathrm{AdS}_{2} \times R^{d}$ was shown to be a solution to Horavava gravity in [9]. If we consider the metric ansatz

$$
d s^{2}=-e(r) d t^{2}+f(r) d r^{2}+d x^{2}+g(x)^{2} d y^{2},
$$

the desired asymptotic behavior on the $\mathrm{AdS}_{2}$, including the length scale $L$, of the metric functions $e(r), f(r)$, and the background scalar potential $\rho(r)$ is given by

$$
e(r) \sim \frac{L^{2}}{r^{2}}, \quad f(r) \sim \frac{L^{2}}{r^{2}}, \quad \rho(r) \sim \frac{Q L}{r},
$$

with the aether vector

$$
u_{M}=\left(-\sqrt{e(r)} \sqrt{\frac{a(r)^{2}+f(r)}{f(r)}}, a(r), 0,0\right) .
$$

Since the asymptotic behavior of the aether vector is to encode AdS time, in the $r \rightarrow 0$ limit $a(r) \sim 0$. Starting from scratch, we insert the ansatz into the equations of motion, and we find $\Lambda=\frac{-2+c_{4}+\kappa Q^{2}}{2 L^{2}}$ is required. This leaves us to solve for $g(x)$, which yields

$$
g(x)=c_{1} e^{\sqrt{1-c_{4}-\kappa Q^{2}} \frac{x}{L}}+c_{2} e^{-\sqrt{1-c_{4}-\kappa Q^{2}} \frac{x}{L}} .
$$

Thus, we see that an $\mathrm{AdS}_{2} \times R^{2}$ can be obtained by choosing $Q= \pm \sqrt{\frac{1-c_{4}}{\kappa}}$ and $\Lambda=-\frac{1}{2 L^{2}}$, resulting in constant $g(x)$. Furthermore, we can observe that for $Q>\sqrt{\frac{1-c_{4}}{\kappa}}$

$$
L_{H}=\frac{L}{\sqrt{1-c_{4}-\kappa Q^{2}}}, \quad \Lambda<-\frac{1}{2 L^{2}}, \quad \mathcal{M}^{2}=H^{2}
$$

where $L_{H}$ is the hyperboloid curvature radius. Additionally, for $Q<\sqrt{\frac{1-c_{4}}{\kappa}}$,

$$
L_{S}=\frac{L}{\sqrt{\left|1-c_{4}-\kappa Q^{2}\right|}}, \quad \Lambda>-\frac{1}{2 L^{2}}, \quad \mathcal{M}^{2}=S^{2}
$$

with $L_{S}$ being the $S^{2}$ curvature radius. In comparing to the $\Lambda=0$ solution found above, we find agreement with the result for $Q$ in eq. (3.8). 
It is important to note that the known analytic black hole solutions of Hořava gravity as presented in $[4,7,10]$ all have this near universal horizon geometry of $\operatorname{AdS}_{2} \times \mathcal{M}^{2}$. This motivates us to explore these geometries with the hope that they describe the emergent near universal horizon behavior of generic black hole solutions.

\section{NR holography on the $\mathrm{AdS}_{2}$}

In this section, we explore the holographic description of non-relativistic, charged scalar fields living in the throat of the geometry (3.6). That is, given the results of [2], we would like to understand the dual non-relativistic $0+1$ dimensional theory to our Hořava solution. Specifically, we can consider two-point correlation functions on $\mathrm{AdS}_{2} \times R^{2}$ as shown above. In doing so, we can compare to the fully relativistic results in [11] to probe the differences between employing holography for fields that respect different boundary symmetries on the same background.

Consider the action of a charged scalar with manifest Schrödinger symmetry [12]

$$
S=\int d t d r d^{2} x \sqrt{G} \frac{N^{2}}{\lambda}\left[\left(\frac{\imath \lambda}{2 N^{2}} \Psi^{\dagger}\left(\mathcal{D}_{t}-N^{J} \mathcal{D}_{J}\right) \Psi+\text { h.c. }\right)-G^{I J} \mathcal{D}_{I} \Psi^{\dagger} \mathcal{D}_{J} \Psi-M^{2} \Psi^{\dagger} \Psi\right]
$$

where in accordance with the analysis done in [2], $\mathcal{D}_{t}=\partial_{t}-\imath \mathcal{A}_{t}$ and $\mathcal{D}_{I}=\nabla_{I}-\imath \mathcal{A}_{I}$ are the gauge covariant derivatives. The background gauge field is $\mathcal{A}_{\mu}=\left(\frac{g}{r}, \overrightarrow{0}\right), G^{I J}$ is the inverse spatial metric, $M$ is the mass of the charged scalars, and $\lambda / 2$ is their charge. $N$ is the lapse encoding global time, and the shift vector $N_{I}$ vanishes in $\mathrm{AdS}_{2} \times R^{2}$.

After Fourier transforming in the orthogonal, $(t, \vec{x})$, directions $\Psi=\psi(r) e^{-\imath \omega t} e^{\imath \vec{k} \cdot \vec{x}}$, the equation of motion for the scalar field $\psi$ is

$$
\psi^{\prime \prime}(r)-\frac{\psi^{\prime}(r)}{r}+\left(\lambda\left(\frac{g}{r}+\omega\right)-\frac{\kappa}{r^{2}}\right) \psi(r)=0,
$$

where $\kappa \equiv k^{2}+M^{2}$. Equation (4.2) can be solved exactly in terms of confluent hypergeometric functions of the second kind, $\mathrm{U}(a ; b ; z)$, and generalized Laguerre polynomials $L(a ; b ; z)$ :

$$
\psi(r)=e^{-i \sqrt{\lambda \omega} r} r^{1+\nu}\left[\widetilde{C}_{1} U(\chi ; 1+2 \nu ; 2 i \sqrt{\lambda \omega} r)+\widetilde{C}_{2} L(-\chi ; 2 \nu ; 2 i \sqrt{\lambda \omega} r)\right],
$$

where $\chi=\frac{1}{2}+\nu+i \frac{g}{2} \sqrt{\frac{\lambda}{\omega}}$ and $\nu \equiv \sqrt{1+\kappa^{2}}$. Making an asymptotic expansion of the solution in the bulk $(r \rightarrow \infty)$, we find that both terms have ingoing and outgoing contributions. To eliminate the outgoing contributions and thereby reconstruct a retarded Green's function, we can rewrite the solution in terms of Whittaker function of the first kind $\mathcal{M}(a ; b ; z)$ :

$$
\psi(r)=C_{1} \sqrt{r} \mathcal{M}\left(-\imath \frac{g}{2} \sqrt{\frac{\lambda}{\omega}} ; \nu ; 2 \imath r \sqrt{\lambda \omega}\right)+C_{2} \sqrt{r} \mathcal{M}\left(-\imath \frac{g}{2} \sqrt{\frac{\lambda}{\omega}} ;-\nu ; 2 \imath r \sqrt{\lambda \omega}\right),
$$

where $C_{1}$ and $C_{2}$ are non-trivially related to the original constants $\widetilde{C}_{1}, \widetilde{C}_{2}$ in (4.3) and generically have $k$ and $\omega$ dependence. While both terms in (4.4) have ingoing and outgoing contributions, the Whittaker function admits an asymptotic expansion $\mathcal{M}(a ; b ; z) \sim$ $e^{-z / 2} F(a, b, z)+e^{z / 2} G(a, b, z)$ that explicitly separates the ingoing and outgoing contributions, and the coefficients $F(a, b, z)$ and $G(a, b, z)$ are given in closed form. 
Restricting (4.4) to be purely ingoing in the bulk fixes the ratio $\frac{C_{2}}{C_{1}}$. The near-boundary behavior $(r \rightarrow 0)$ of $\psi$ is then

$$
\psi(r \rightarrow 0) \sim A(\omega, k) r^{\Delta_{-}}+B(\omega, k) r^{\Delta_{+}},
$$

where $\Delta_{ \pm}=1 \pm \nu$ sets the scaling dimension of the dual operator $\Delta=\Delta_{+}$. The retarded Green's function of the dual theory is then

$$
G_{R}(\omega, k)=\frac{B(\omega, k)}{A(\omega, k)}=e^{-2 \pi \imath \nu} \frac{\Gamma(-2 \nu) \Gamma\left(\frac{1}{2}+\nu-\imath \frac{g}{2} \sqrt{\frac{\lambda}{\omega}}\right)}{\Gamma(2 \nu) \Gamma\left(\frac{1}{2}-\nu-\imath \frac{g}{2} \sqrt{\frac{\lambda}{\omega}}\right)}(2 \imath \sqrt{\lambda \omega})^{2 \nu},
$$

following $[11,13]$. While there are apparent similarities between eq. (4.6) and the two-point functions calculated in $[11,13]$, we should note the fundamental differences that reflect the non-relativistic nature of the theory we are considering.

The $\nu$ that appears here is strictly real and positive, while for relativistic charged scalars, $\nu$ can take imaginary values as the gauge coupling increases. The interpretation given by the authors in [11] was that sufficiently strong electric fields induced pair production of the charged scalars. Our results fit consistently within this interpretation as, for the non-relativistic theory, we do not see such behavior. Since $\nu$ does not become imaginary with large $g$, we find that

$$
\lim _{g \rightarrow \infty} G_{R}(\omega, k)=e^{-2 \pi \imath \nu} \frac{\Gamma(-2 \nu)}{\Gamma(2 \nu)}(g \lambda)^{2 \nu} .
$$

In particular we find that $G_{R}$ becomes independent of the frequency $\omega$. We will further discuss this feature below. Note, as we turn off the background electric field $g \rightarrow 0$, eq. (4.6) becomes

$$
\lim _{g \rightarrow 0} G_{R}(\omega, k)=e^{-2 \pi \imath \nu} \frac{\Gamma(-2 \nu)}{\Gamma(2 \nu)}\left(\frac{\imath}{2} \sqrt{\lambda \omega}\right)^{2 \nu} .
$$

This recovers the known power law behavior for the two-point function for non-relativistic scalars, i.e., $G_{R}(\omega, k) \sim \omega^{\nu}[14]$.

The prefactors of $\omega^{\nu}$ appearing in eq. (4.6) possess a non-trivial dependence on the frequency. In particular, the combination $g \sqrt{\frac{\lambda}{\omega}}$ in the Gamma functions indicates the emergence of a new scale in the non-relativistic dual theory, which is not found in the relativistic theory considered in $[11,13]$. This may be expected, as the gauge coupling $g$ scales as a velocity, which becomes a dimensionful quantity in the non-relativistic theory. ${ }^{1}$ It may be useful to think of $g$ as setting a velocity scale analogous to a Fermi velocity in the near-horizon limit. Then the $\omega$-independence of (4.7) suggests that the response of the scalar field on the boundary becomes instantaneous in the large velocity limit. Whether this analogy between $g$ and the presence of a Fermi surface can be made more precise is an open question.

\footnotetext{
${ }^{1}$ In relativistic theories we are used to fixing units so that the two fundamental constants $\hbar=c=1$. In these natural units length and time are both measured in inverse $\mathrm{eV}$, and so velocities are pure numbers. In the non-relativistic Schrödinger equation $c$ does not appear; instead one commonly fixes units so that $\hbar=m=1$, where $m$ is the particle mass. Since $E \sim m v^{2}$, this now implies that velocity squared divided by energy is a dimensionless combination, not velocity by itself.
} 


\section{Conclusion}

In this work, we have explored the role that charged backgrounds play in the khronometric formulation of Hořava gravity through static, spherically symmetric Einstein-Æther theory. We have found that the causal boundaries appearing in Schwarzschild-type black holes, both asymptotically flat and AdS, are present in the AdS-Reissner-Nördstrom background as well. This provides yet more evidence that universal horizons are indeed a generic feature of the probe limit of khronometric Hořava gravity. An analytic solution for a charged black hole was also constructed in section 3 where we found that an asymptotically flat extremal Reissner-Nördstrom geometry solved the full equations of motion for the metric functions, æther vector, and the scalar potential. Given the existence of universal horizons in the probe limit of Hořava an important question is the nature of their stability. It is argued in [6] that although high multipole finite speed modes of the khronon are stable, the universal horizon is unstable to perturbations of the instantaneous mode of the khronon. Such instabilities may be removed by higher derivative terms in the full Hořava action. These issues are important in understanding the thermodynamics of universal horizons and possible holographic applications, and therefore deserve deeper study.

In exploring the near horizon $\mathrm{AdS}_{2} \times R^{2}$ emergent geometry, we have calculated the two point retarded Green's function for non-relativistic charged scalars. We have found that there is a striking similarity to the relativistic results that have been found previously, which may have been anticipated given the nature of the Hořava solution we have found. There are, however as of yet, unresolved issues with the results obtained in section 4 . In the relativistic calculations, it was natural to regard the dual description of the near horizon geometry as a CFT emerging due to zooming in on a local section of a fractional Fermi surface. It is unclear whether this language can be faithfully imported to the nonrelativistic theory as we have an additional scale present, which could in this vernacular be interpreted as the Fermi velocity. We will leave the resolution of this question for future exploration.

\section{Acknowledgments}

We would like to thank Michael Wagman for conversations during the early stages of this work. This work was supported in part by the U.S. Department of Energy under Grant No. DE-FG02-96ER40956. 
Open Access. This article is distributed under the terms of the Creative Commons Attribution License (CC-BY 4.0), which permits any use, distribution and reproduction in any medium, provided the original author(s) and source are credited.

\section{References}

[1] P. Hořava, Quantum Gravity at a Lifshitz Point, Phys. Rev. D 79 (2009) 084008 [arXiv:0901.3775] [INSPIRE].

[2] S. Janiszewski and A. Karch, Non-relativistic holography from Hoŕava gravity, JHEP 02 (2013) 123 [arXiv:1211.0005] [INSPIRE].

[3] D. Blas, O. Pujolàs and S. Sibiryakov, Models of non-relativistic quantum gravity: the good, the bad and the healthy, JHEP 04 (2011) 018 [arXiv: 1007.3503] [INSPIRE].

[4] P. Berglund, J. Bhattacharyya and D. Mattingly, Mechanics of universal horizons, Phys. Rev. D 85 (2012) 124019 [arXiv: 1202.4497] [InSPIRE].

[5] T. Jacobson, Undoing the twist: the Hor̆ava limit of Einstein-aether, arXiv:1310.5115 [INSPIRE].

[6] D. Blas and S. Sibiryakov, Hoŕava gravity versus thermodynamics: the black hole case, Phys. Rev. D 84 (2011) 124043 [arXiv:1110.2195] [INSPIRE].

[7] S. Janiszewski, Asymptotically hyperbolic black holes in Hor̆ava gravity, arXiv:1401.1463 [INSPIRE].

[8] P. Hořava and C.M. Melby-Thompson, General Covariance in Quantum Gravity at a Lifshitz Point, Phys. Rev. D 82 (2010) 064027 [arXiv: 1007.2410] [InSPIRE].

[9] M. Alishahiha and H. Yavartanoo, Conformally Lifshitz solutions from Hořava-Lifshitz Gravity, arXiv: 1212.4190 [INSPIRE].

[10] J. Bhattacharyya, Aspects of holography in Lorentz-violating gravity, Ph.D. Thesis, University of New Hampshire, U.S.A. (2013).

[11] T. Faulkner, H. Liu, J. McGreevy and D. Vegh, Emergent quantum criticality, Fermi surfaces and $A d S_{2}$, Phys. Rev. D 83 (2011) 125002 [arXiv:0907.2694] [INSPIRE].

[12] D.T. Son, Toward an AdS/cold atoms correspondence: a geometric realization of the Schrödinger symmetry, Phys. Rev. D 78 (2008) 046003 [arXiv: 0804.3972] [INSPIRE].

[13] T. Faulkner, N. Iqbal, H. Liu, J. McGreevy and D. Vegh, Holographic non-Fermi liquid fixed points, Phil. Trans. Roy. Soc. A 369 (2011) 1640.

[14] K. Balasubramanian and J. McGreevy, Gravity duals for non-relativistic CFTs, Phys. Rev. Lett. 101 (2008) 061601 [arXiv:0804.4053] [INSPIRE]. 